CASE REPORT

\title{
The elevated serum alkaline phosphatase - the chase that led to two endocrinopathies and one possible unifying diagnosis
}

Hsu Li-Fern and Rajasoorya C

Department of Medicine, Alexandra Hospital, Singapore

(Correspondence should be addressed to Rajasoorya C, Department of Medicine, Alexandra Hospital, 378 Alexandra Road, Singapore 159964)

\begin{abstract}
A 39-year-old Chinese man with hypertension being evaluated for elevated serum alkaline phosphatase (SAP) levels was found to have an incidental right adrenal mass. The radiological features were characteristic of a large adrenal myelolipoma. This mass was resected and the diagnosis confirmed pathologically. His blood pressure normalised after removal of the myelolipoma, suggesting that the frequently observed association between myelolipomas and hypertension may not be entirely coincidental. Persistent elevation of the SAP levels and the discovery of hypercalcaemia after surgery led to further investigations which confirmed primary hyperparathyroidism due to a parathyroid adenoma. The patient's serum biochemistry normalised after removal of the adenoma.

The association of adrenal myelolipoma with primary hyperparathyroidism has been reported in the literature only once previously. Although unconfirmed by genetic studies this association may possibly represent an unusual variation of the multiple endocrine neoplasia type 1 syndrome.
\end{abstract}

European Journal of Endocrinology 140 143-147

\section{Introduction}

Myelolipomas of the adrenal gland are benign tumours composed of mature adipose and haemopoietic tissue. They are very rare, with an incidence rate of between 0.08 to $0.20 \%$ at autopsy (1), and are usually hormonally inactive $(1,2)$. Often clinically silent, most cases are discovered at autopsy. The majority of the rest appear as incidental findings during abdominal imaging procedures, forming about $7-15 \%$ of all incidentally discovered adrenal masses (3).

We describe a patient with an incidentally discovered adrenal myelolipoma who was also found to have primary hyperparathyroidism due to a parathyroid adenoma, and we discuss the syndromic differential diagnosis of this unusual association.

\section{Case report}

A 39-year-old Chinese man, who was initially investigated for suspected hepatitis, was referred to our hospital for investigation of isolated persistently elevated serum alkaline phosphatase (SAP) levels, which ranged from 140 to $199 \mathrm{U} / \mathrm{l}$ (normal range 40-130 U/l) on repeated testing. Other tests of liver function were normal. He had also complained of lethargy, paroxysmal sweating and intermittent headaches for a few months, and had a history of hypertension since the age of 35 which was satisfactorily controlled with Nifedipine. Investigations carried out at that time to exclude secondary causes of hypertension, including serum electrolytes, urinalysis and a chest radiograph were normal. Urine vanillylmandelic acid (VMA) and catecholamine levels then were within the normal range. A subsequent intravenous urogram (IVU) performed a year prior to the present consultation for investigation of renal colic was also reported to be normal, although differential lengths of the right and left kidneys of 11.5 and $14.0 \mathrm{~cm}$ respectively were noted.

Physical examination was unremarkable, and blood counts and serum electrolytes including potassium were normal. In view of the recent history of hypertension and symptoms of paroxysmal sweating and headaches, investigations for phaeochromocytoma were repeated. However, his urine VMA and catecholamine levels were once again normal.

Abdominal ultrasonography performed to exclude a hepatic cause of his raised SAP levels revealed a large, homogenously echogenic mass below the right lobe of the liver and above the upper pole of the right kidney. A subsequent computed tomography (CT) scan showed a large $10 \times 6 \mathrm{~cm}$ non-enhancing mass in the right suprarenal area with attenuation consistent with adipose tissue (Fig. 1). However, there was still doubt 


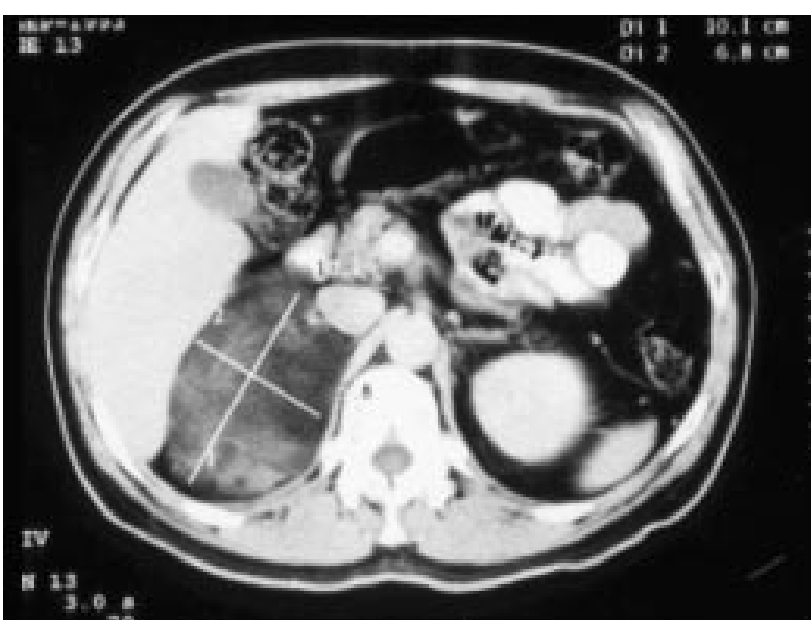

Figure $1 \mathrm{CT}$ scan of the abdomen showing the large non-enhancing mass in the right suprarenal space.

as to whether the mass was of renal or adrenal origin. Consequently, the patient underwent angiography, which revealed a sizeable hypovascular right adrenal tumour with commensurate inferior displacement of the right kidney (Fig. 2). Post angiography the radiologist performed an unplanned simultaneous adrenal vein sampling for renin and aldosterone. With certainty of catheter placement, the plasma aldosterone (PA) levels were measured as $>3300 \mathrm{pmol} / \mathrm{l}$ in the right adrenal vein and $1350 \mathrm{pmol} / \mathrm{l}$ in the left adrenal vein. Aldosterone measurements from the right adrenal vein were not done in further dilutions. The simultaneous

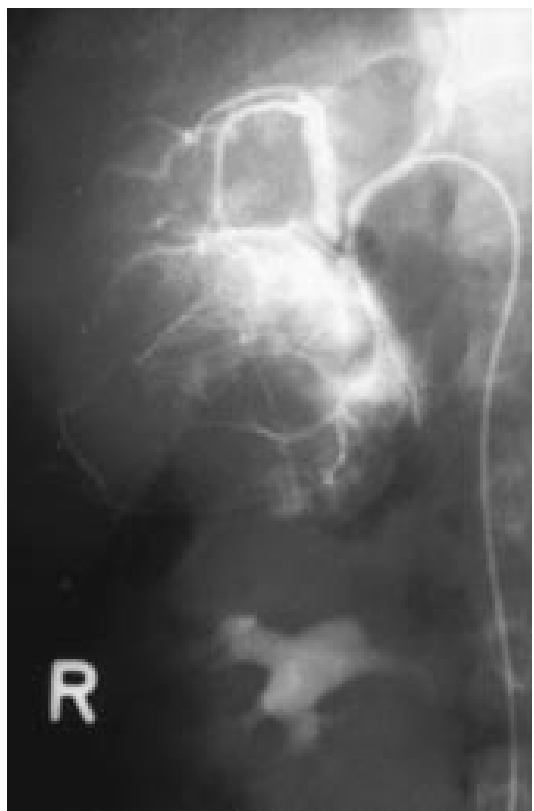

Figure 2 Angiogram showing a hypovascular tumour arising from the right adrenal gland with associated renal compression. peripheral PA levels were 134-232 pmol/l (normal values $16.6-617.7 \mathrm{pmol} / \mathrm{l}$ ). Plasma renin activitiy (PRA) measured in all samples during the study were between $0.09-0.34 \mathrm{pg} / \mathrm{l} / \mathrm{h}$ (normal peripheral supine $\mathrm{PRA}=0.66-3.08 \mathrm{pg} / \mathrm{l} / \mathrm{h})$. The PA/PRA ratios in the peripheral samples were not elevated. On retrospective reassessment, the intravenous pyelogram performed the previous year revealed a large right suprarenal mass, which had been missed when the films were reported as normal (Fig. 3, upper panel). Compression of the kidney could have resulted in the differential renal sizes noted. From the sonographic, CT and angiographic information, a diagnosis of adrenal myelolipoma was made.

Because of the large size of the mass, radiological evidence of renal and hepatic compression, and the need to exclude an aldosteronoma causing the suppressed renin levels or an intermittently secreting phaeochromocytoma in view of his symptoms, right adrenalectomy was performed under standard precautions as for a phaeochromocytoma. Macroscopically, the specimen was an $11 \times 9 \times 5 \mathrm{~cm}$ encapsulated mass containing lipomatous and haemorrhagic areas, surrounded by a thin rim of yellowish tissue. Microscopically, the sections showed mainly mature adipose tissue with areas of haemorrhage and foci of haemopoietic cells composed of both the erythroid and myeloid series, surrounded by a rim of adrenocortical tissue and bound by a capsule. This confirmed the diagnosis of an adrenal myelolipoma. No aldosteronoma or abnormalities of adrenocortical tissue were demonstrable on pathology.
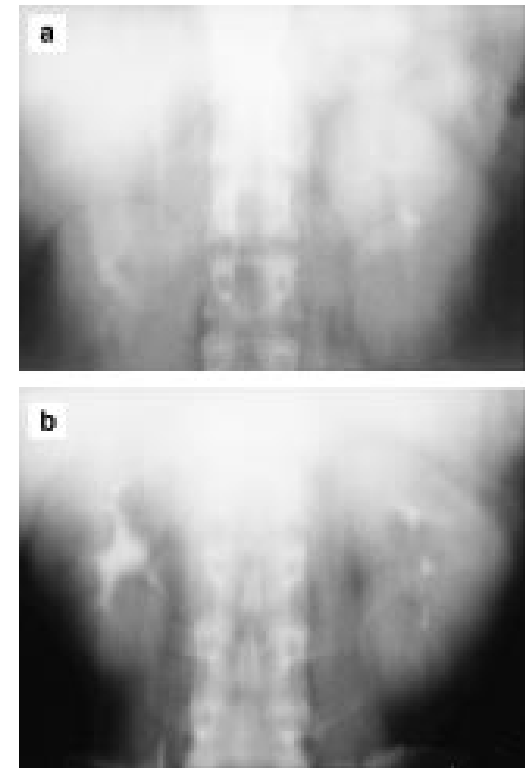

Figure 3 (Upper panel) The IVU prior to the discovery of the myelolipoma. Note the differential sizes of the kidneys, and the faint outline of a mass above the right kidney, which was missed initially. (Lower panel) The second IVU, performed after resection of the myelolipoma, was normal. 
Post-operatively, the patient did not develop hypotension, but his blood pressure subsequently normalised and he no longer required anti-hypertensive medication. However, his SAP levels remained elevated, and in addition to his previous symptoms, he also began to complain of polydipsia and episodes of unexplained abdominal colic. A second IVU performed to investigate the abdominal colic was normal, and there was no discrepancy in kidney size, the right kidney having returned to its normal size and contour (Fig. 3, lower panel). Further investigations revealed elevated fractions of bone-specific alkaline phosphatase, as well as hypercalcaemia and hypophosphataemia. His corrected serum calcium level was $3.41 \mathrm{mmol} / \mathrm{l}$ (normal 2.05$2.65 \mathrm{mmol} / \mathrm{l}$ ) while his phosphate level was $0.80 \mathrm{mmol} / \mathrm{l}$ (normal $0.85-1.45 \mathrm{mmol} / \mathrm{l}$ ). Parathyroid hormone (PTH) level was $132.9 \mathrm{pmol} / \mathrm{l}$ (normal $0.5-5.1 \mathrm{pmol} / \mathrm{l}$ ), confirming the diagnosis of primary hyperparathyroidism. Magnetic resonance imaging studies of the neck revealed a tumour of the right inferior parathyroid gland, which was excised surgically. Histologically, the $2 \times 1.2 \times 0.3 \mathrm{~cm}$ tumour was confirmed to be a parathyroid adenoma, composed predominantly of chief cells. The other parathyroid glands were normal on inspection.

After the second operation, from which he recovered without complications, the patient's serum calcium, phosphate, SAP and PTH levels all reverted to normal. All his symptoms resolved and he has remained well on subsequent visits over more than a year. Follow-up questioning did not elicit a family history of hyperparathyroidism or other endocrine conditions, and his family members were reluctant to come for screening tests.

\section{Discussion}

This case illustrates, first of all, the need to consider all possible differential diagnoses when faced with an abnormal finding or result. In our patient, the first error was the failure to notice the myelolipoma on the initial IVU. A suprarenal mass can be seen faintly above the right kidney on closer inspection of the films. In addition, the normal kidney size on an IVU is 10$16 \mathrm{~cm}$, with the difference in length of both kidneys usually not more than $1.5 \mathrm{~cm}$. In our patient, the difference of $2.5 \mathrm{~cm}$, together with the uneven contour of the superior pole of the right kidney, should have raised our suspicions of a possible suprarenal mass. In retrospect, the features are striking, especially when viewed alongside his second IVU which was performed after removal of the myelolipoma (Fig. 3).

The second error was the assumption that the elevated SAP levels were of hepatic origin and hence no calcium or bone studies were performed. In retrospect, his presenting symptoms of lethargy, sweating and headache could have been caused by hypercalcaemia, and hence should have been given due consideration. As it was, the resultant investigations for hepatic disease led to the incidental discovery and surgical removal of the right adrenal myelolipoma. However, the primary problem of raised SAP was still not resolved until the discovery of primary hyperparathyroidism and the removal of the parathyroid adenoma much later.

It can be argued that because of the failure to consider calcium studies, the patient was subjected to unnecessary investigations, and that removal of the incidentally discovered myelolipoma, which involved added surgical risk, was of little consequence as he was asymptomatic. It is possible that if calcium studies had been performed early and hyperparathyroidism diagnosed and treated, the myelolipoma would have remained undiscovered. However, it has been recommended in the literature that large myelolipomas be removed to prevent possible serious complications like retroperitoneal haemorrhage $(1,2,4)$, and we believe that the errors, in the final analysis, probably benefited the patient. The cure of the hypertension was an additional benefit sustained by the patient as a consequence of the sequential errors.

Myelolipomas are benign tumours that exhibit variable growth (5). In our patient, it appeared to be relatively slow growing, as it could already be seen (but was missed) in the IVU performed more than a year prior to its discovery. Although usually asymptomatic, they can cause abdominal pain and compression symptoms, especially when they become large, necrotic or haemorrhagic $(1,2,4,6)$. Diagnosis can often be made radiologically, as they have characteristic findings. As seen in our patient, ultrasonography usually reveals hyperechogenic tissue with propagation speed artifact, while CT scans will reveal lesions with the attenuation of adipose tissue $(5,7)$. If doubt persists, especially with regard to possible malignancy, further information can be obtained by angiography, which will show a hypovascular tumour (2), and CT-guided fine needle aspiration biopsy for tissue diagnosis (8).

The management of adrenal myelolipoma is individualised. It has been recommended that surgery should be reserved only for symptomatic tumours as well as asymptomatic large tumours more than $4 \mathrm{~cm}$ in size, as found in our patient. These large tumours are associated with a higher risk for retroperitoneal haemorrhage $(1,2,9)$. Small tumours can be monitored clinically with periodic ultrasound or CT examination $(1,9)$.

Various medical conditions have been associated with adrenal myelolipoma, including obesity, various cancers, diabetes mellitus and hypertension $(4,6)$. Although it has been argued that these associated conditions may be entirely coincidental due to their high prevalence in the population (4), it is interesting to note that the blood pressure in our patient normalised after removal of the tumour. 
We considered two possibilities why this patient could have developed hypertension from the adrenal myelolipoma. First, the large size of this tumour could have caused superior renal vasculature compression with resultant hypertension, and this phenomenon has been documented in the literature (6). The second interesting possibility that must be considered is a source of aldosterone excess from the right adrenal gland. Aldosterone overproduction from co-existent adrenocortical hyperplasia (10) has been recorded uncommonly in the literature. It is unfortunate that the aldosterone measurements from the right adrenal vein were not done in further dilutions. As highlighted by Doppman and Gill (11), unilateral hyperaldosteronism is usually associated with a greater than 10-fold increase in aldosterone concentration in the ipsilateral side compared with the contralateral side. In this patient, the absence of a clearly demonstrable elevated aldosterone gradient would be against the possibility of a small and occult aldosteronoma. Even then, no aldosteronoma or zona glomerulosa hyperplasia was noted on careful histolopathological evaluation of the resected specimen. The absence of an elevated PA/PRA ratio in the peripheral blood samples would also be against the diagnosis of primary aldosteronism (12-15). Thus, we postulate that the hypertension was more likely to be due to reno-vascular compression than a concomitant primary aldosteronism state. Although hypertension has been reported to be associated with up to $60 \%$ of patients with hyperparathyroidism (16), we believe that this was not the case in our patient, as he was already normotensive before removal of the parathyroid adenoma.

Other than primary hyperaldosteronism, various other endocrinopathies have also been associated with myelolipomas. These are usually the result of co-existing adrenal tumours or adrenal gland hyperplasia and include Cushing's syndrome, Addison's disease, congenital 21-hydroxylase deficiency $(4,6,10)$ and a case of phaeochromocytoma (17). Although the tumour itself is hormonally inactive by definition, a recent report described foci of active adrenal tissue within a myelolipoma producing subclinical Cushing's syndrome, thus lending a functional component to it (18).

Extra-adrenal endocrine dysfunction has also been reported with myelolipomas, with rare cases of pituitary Cushing's disease and a case of adrenogenital syndrome associated with an interstitial tumour of the testes (10). An unusual case of multiple adrenocortical tumours associated with primary hyperparathyroidism and tumours of the pituitary, pancreas and thyroid has been reported in the context of the multiple endocrine neoplasia type 1 (MEN 1) syndrome (19). The adrenal tumours consisted of a myelolipoma, an adenoma and a carcinoma, and this was the first case where a myelolipoma was found in association with primary hyperparathyroidism.
The possibility that our patient had a variant of one of the MEN syndromes was considered. MEN 1 is a genetically determined syndrome characterised by the combination of tumours arising from the parathyroids, endocrine pancreas and anterior pituitary gland. Primary hyperparathyroidism is by far the most common manifestation, affecting about $95 \%$ of patients $(16,20)$. Adrenocortical, thyroid, carcinoid and lipomatous tumours have also been described (20). Although not considered a major feature of MEN 1, adrenocortical lesions were encountered in 36\% to $40 \%$ of patients with MEN 1 in several clinical series $(21,22)$. A broad range of lesions has been encountered, including diffuse cortical hyperplasia, nodular hyperplasia, adenomas and, rarely, carcinomas. They are occasionally functional, and have been observed to produce Cushing's syndrome (21) and primary hyperaldosteronism (23).

Genetic testing has become increasingly important in the diagnosis and characterisation of MEN 1. The MEN 1 gene maps to chromosome 11q13, and abnormalities can be detected through linkage analysis of DNA or loss of heterozygosity (LOH) studies in the tumours $(24,25)$. A recent analysis of various tumours found in subjects with MEN 1 revealed 11q13 LOH in pituitary tumours, lung carcinoids and lipomas but not in skin angiofibromas, an oesophageal leiomyoma nor a renal angiomyolipoma (26). This appeared to suggest that the MEN 1 gene was responsible for the pathogenesis of some tumours but not others. Unfortunately, genetic testing is not yet widely available. Where available and if willing, it would be of interest and benefit to do genetic studies in our index case and his family members.

A possible syndromic differential diagnosis is the primary hyperparathyroidism and jaw tumour (HPT-JT) syndrome, which has been found to be an independent genetic syndrome with its gene located on chromosone 1q21-q31 (27). It is characterised by the presence of hyperparathyroidism, usually due to parathyroid adenoma or carcinoma, in association with various tumours of the jaw. Various visceral tumours or cysts have been reported in association with this syndrome, including renal harmatomas, cystic kidney disease and multiple uterine myomata $(28,29)$. However, our patient does not fit this syndrome as there was no evidence of jaw tumours.

While the exact aetiology and pathogenesis of adrenal myelolipomas remain unclear, it is widely accepted that they originate from the adrenal cortex $(4,6,19)$, and hence fit the MEN 1 phenotype. To the best of our knowledge, the association of an adrenal myelolipoma and primary hyperparathyroidism has been reported in the literature only once previously (19). Our patient may be only the second such case, and, although unconfirmed by genetic studies, may also possibly represent an unusual variant of the MEN 1 syndrome. 


\section{References}

1 Dieckmann KP, Hamm B, Pickartz H, Jonas D \& Bauer HW. Adrenal myelolipoma: clinical, radiologic and histologic features. Urology 198729 1-8.

2 E-Mekresh MM, Abdel-Gawad M, El-Diasty T, El-Baz M \& Ghoneim MA. Clinical, radiological and histological features of adrenal myelolipoma: review and experience with a further eight cases. British Journal of Urology 199678 345-350.

3 Kloos RT, Gross MD, Francis IR, Korobkin M \& Shapiro B. Incidentally discovered adrenal masses. Endocrine Reviews 1995 16 460-484.

4 Han M, Burnett AL, Fishman EK \& Marshall FF. The natural history and treatment of adrenal myelolipoma. Journal of Urology 1997157 1213-1216.

5 Sanders R, Bissada N, Curry N \& Gordon B. Clinical spectrum of adrenal myelolipoma: analysis of 8 tumours in 7 patients. Journal of Urology $19951531791-1793$

6 Meaglia JP \& Schmidt JD. Natural history of an adrenal myelolipoma. Journal of Urology 1992147 1089-1090.

7 Musante F, Derchi LE, Zappasodi F, Bazzocchi M, Riviezzo GC, Banderali A et al. Myelolipoma of the adrenal gland: sonographic and CT features. American Journal of Roentgenology 1988151 961-964.

8 Dunphy $\mathrm{CH}$. Computed tomography-guided fine needle aspiration biopsy of adrenal myelolipoma. Case report and review of the literature. Acta Cytologica 199135 353-356.

9 Del Gaudio A, Solidoro G \& Del Gaudio GA. Myelolipoma of the adrenal gland. Report on two cases. European Journal of Surgery $1992158629-632$.

10 Oliva A, Duarte B, Hammadeh R, Ghosh L \& Baker RJ. Myelolipoma and endocrine dysfunction. Surgery 1988103 711-715.

11 Doppman JL \& Gill JR. Hyperaldosteronism. Sampling the renal veins. Radiology 1996198 309-312.

12 Eng PHK, Tan KEK, Khoo DHC, Tan CE, Lim HS, Lim SC et al. Aldosterone to renin ratios in the evaluation of primary aldosteronism. Annals of the Academy of Medicine of Singapore $199726762-766$.

13 Gordon RD. Mineralocorticoid hypertension. Lancet 1994344 240-243.

14 Weinberger MH \& Fineberg NS. The diagnosis of primary aldosteronism and separation of two major subtypes. Archives of Internal Medicine 1993153 2125-2129.

15 McKenna TJ, Sequeira SJ, Heffernan A, Chambers J \& Cunningham S. Diagnosis under random conditions of all disorders of the renin-angiotensin-aldosterone axis, including primary hyperaldosteronism. Journal of Clinical Endocrinology and Metabolism 199173 952-957.

16 Cleland SJ \& Connell JMC. Endocrine hypertension. Journal of the Royal College of Physicians of London 199832 104-108.

17 Ukimura O, Inui E, Ochiai A, Kojima M \& Watanabe H. Combined adrenal myelolipoma and phaeochromocytoma. Journal of Urology 19951541470 .
18 Boronat M, Moreno A, Ramon y Cajal S, Pineda E, Lucas T \& Estrada J. Subclinical Cushing's syndrome due to adrenal myelolipoma. Archives of Pathology and Laboratory Medicine 1997 $121735-737$.

19 Banik S, Hasleton PS \& Lyon RL. An unusual variant of multiple endocrine neoplasia syndrome: a case report. Histopathology $19848135-144$.

20 Raue F \& Zink A. Clinical features of multiple endocrine neoplasia type 1 and type 2. Hormone Research 199238 (Suppl 2) 31-35.

21 Burgess JR, Harle RA, Tucker P, Parameswaran V, Davies P, Greenaway TM et al. Adrenal lesions in a large kindred with multiple endocrine neoplasia type 1. Archives of Surgery 1996131 699-702.

22 Skogseid B, Rastad J, Gobl A, Larsson C, Backlin K, Juhlin C et al. Adrenal lesion in multiple endocrine neoplasia type 1. Surgery $19951181077-1082$

23 Ferris JB, Brown JJ, Cumming AM, Fraser R, Lever AF, Peacock M et al. Primary hyperparathyroidism associated with primary hyperaldosteronism. Acta Endocrinologica 1983103 365-370.

24 Calender A, Giraud S, Schuffenecker I, Lenoir GB, Caudray P, Courseaux A et al. Genetic testing in presymptomatic diagnosis of multiple endocrine neoplasia. Hormone Research 199747 199-210.

25 Marx S, Spiegel AM, Skarulis MC, Doppman JL, Collins FS \& Liotta LA. Multiple endocrine neoplasia type 1: clinical and genetic topics. Annals of Internal Medicine $1998129484-494$.

26 Dong Q, Debelenko LV, Chandrasekharappa SC, Emmert-Buck MR, Zhuang Z, Guru SC et al. Loss of heterozygosity at 11q13: analysis of pituitary tumors, lung carcinoids, lipomas, and other uncommon tumors in subjects with familial multiple endocrine neoplasia type 1. Journal of Clinical Endocrinology and Metabolism $1997821416-1420$.

27 Szabo J, Heath B, Hill VM, Jackson CE, Zarbo RJ, Mallette LE et al. Hereditary hyperparathyroidism-jaw tumour syndrome: the endocrine tumour gene HRPT2 maps to chromosome 1q21q31. American Journal of Human Genetics 199556 944-950.

28 Teh BT, Farnebo F, Kristoffersson U, Sundelin B, Cardinal J, Axelson $\mathrm{R}$ et al. Autosomal dominant primary hyperparathyroidism and jaw tumour syndrome associated with renal harmatomas and cystic renal disease: linkage to 1q21-q32 and loss of wild type allele in renal harmatomas. Journal of Clinical Endocrinology and Metabolism 199681 4204-4211.

29 Fujikawa M, Okamura K, Sato K, Mizokami T, Tamaki K, Yanagida $\mathrm{T}$ et al. Familial isolated hyperparathyroidism due to multiple adenomas associated with ossifying jaw fibroma and multiple uterine adenomyomatous polyps. European Journal of Endocrinology 1998138 557-561.

Received 23 October 1998

Accepted 26 October 1998 